\title{
Application of Monte Carlo Method for Evaluation of Uncertainties of ITS-90 by Standard Platinum Resistance Thermometer
}

\author{
Rudolf Palenčár, Peter Sopkuliak, Jakub Palenčár, Stanislav Ďuriš, Emil Suroviak, Martin Halaj \\ Faculty of Mechanical Engineering, Institute of Automation, Measurements and Applied Informatics, Slovak Technical \\ University, 81243 Bratislava, Nám. Slobody 17, SlovakRepublic,email: rudolf.palencar@stuba.sk, xsopkuliak@is.stuba.sk
}

\begin{abstract}
Evaluation of uncertainties of the temperature measurement by standard platinum resistance thermometer calibrated at the defining fixed points according to ITS-90 is a problem that can be solved in different ways. The paper presents a procedure based on the propagation of distributions using the Monte Carlo method. The procedure employs generation of pseudo-random numbers for the input variables of resistances at the defining fixed points, supposing the multivariate Gaussian distribution for input quantities. This allows taking into account the correlations among resistances at the defining fixed points. Assumption of Gaussian probability density function is acceptable, with respect to the several sources of uncertainties of resistances. In the case of uncorrelated resistances at the defining fixed points, the method is applicable to any probability density function. Validation of the law of propagation of uncertainty using the Monte Carlo method is presented on the example of specific data for $25 \Omega$ standard platinum resistance thermometer in the temperature range from 0 to $660{ }^{\circ} \mathrm{C}$. Using this example, we demonstrate suitability of the method by validation of its results.
\end{abstract}

Keywords: The law of propagation of uncertainty, Monte Carlo method, the International Temperature Scale of 1990 (ITS-90), Standard Platinum Resistance Thermometer.

\section{INTRODUCTION}

This paper presents a method based on the propagation of distributions by Monte Carlo method (MCM). The procedure is based on the generation of pseudo-random numbers of input variables of multi-dimensional distribution. Multi-dimensional distribution is used because it takes into account correlation among the Standard Platinum Resistance Thermometer (SPRT) resistances from calibration as well as the SPRT resistances in temperature measurement. Generating input variables only from the onedimensional distribution is sufficient for uncorrelated resistances.

In our case it is necessary to identify the probability distributions of input quantities and relevant multivariate distribution function for the case of correlated input quantities. We can assume normal distribution for all input SPRT resistances and therefore multivariate normal distribution for correlated resistances. This assumption is based on the central limit theorem, because several sources of uncertainties are present at the measurement: e.g. selfheating effect of the SPRT, chemical impurities of the substance in defining fixed points (DFPs), immersion effect of the SPRT, hydrostatic-head effect, effect of gas pressure in DFPs, choice of fixed point value from plateau isotopic variations, residual gas pressure in triple point of water
(TPW) cell, non-linearity of the resistance bridge, changes of resistances of standard resistor initiated by changes of its temperature, uncertainty of calibration of resistance standard, etc.

The aim of this study is

a) presentation of the MCM for uncertainty evaluation of the international temperature scale ITS-90 by using SPRT calibrated at DFPs;

b) validation of the process by using the law of propagation of uncertainty according to the Guide to the Expression of Uncertainty in Measurement GUM [1] for specific conditions.

The procedure was designed to take into account correlations among the SPRT resistances obtained from calibration at the DFPs and those obtained in temperature measurements.

Influences such as fluctuations, drifts, temperature gradients and similar are not analyzed. Also, the uncertainties caused by non-uniqueness and consistency sub-ranges are not included. These questions are presented e.g. in [2]. The case study focuses on determination of uncertainties related to realization of international temperature scale by using SPRT calibrated in the range from $0{ }^{\circ} \mathrm{C}$ to the freezing point of aluminum $\left(660.323^{\circ} \mathrm{C}\right)$. 


\section{Current Status of THE ISSUE}

Most published papers employ an approach based on the law of propagation of uncertainty GUM and its supplements [3] and [4], fewer papers are based on the orthogonal polynomials. The overview of the approaches is presented in [5], [6]. Matrix interpretation of GUM method is described in [7]. Specific approaches dealing with density functions and confidence intervals are mentioned in [8], [9]. The effect of covariance on uncertainty when realizing ITS-90 temperature scale is discussed in [6]. Uncertainty determination by $\mathrm{MCM}$ is discussed e.g. in [10], [11]. Propagation of distributions using MCM, based on Supplement 1 [3] to the GUM [1], occurs only in a few isolated cases e.g. [12], [13]. In general, authors predict uncorrelated resistances among the defining fixed point (DFP) and neglect the influence of correlations. Omitting the correlations among resistances in individual DFPs does not always correspond to reality and they can have a significant impact, see [6]. Progressive Bayesian analysis introduces another point of view. Simple measurement model is presented in [14].

Deviation equations and ratios of resistance belong to a non-linear model, defined by ITS-90. The non-linear model can generate some doubts about the adequacy of its linearization by expansion in Taylor series of the first order. Also, complications with the determination of the sensitivity coefficients may occur. In such cases, Monte Carlo method, based on propagation of distributions, is preferably used. Papers on the Monte Carlo method, which follows the recommendations and procedures listed in [3], appear sporadically. The basic principle of both methods is shown in Fig. 1.

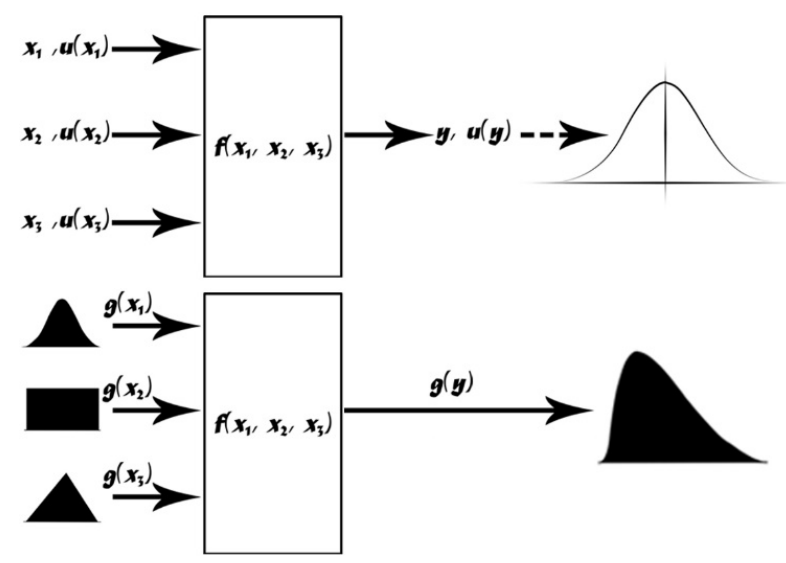

Fig.1. The law of propagation of uncertainty (up) and the law of propagation of distribution (down).

\section{THEORETICAL BASES OF ITS-90}

International Temperature scale of 1990 defines the temperature from the inverse function

$$
T=f\left(W_{r}\right)
$$

Corresponding sub-ranges of the ITS-90 from $0{ }^{\circ} \mathrm{C}$ for the functions (1) are stated in [15]. Function $W_{r}$ is given by

$$
W_{r}=W-\sum_{i=1}^{N} a_{i} f_{i}(W)
$$

where

$$
W=\frac{R}{R_{\mathrm{TPW}}}
$$

while

$R$ is the SPRT resistance at temperature $T$ and $R_{\mathrm{TPW}}$ is the SPRT resistance at TPW

$f_{i}(W)$ are functions of the individual sub-ranges, see $[15]$.

$a_{i}$ are coefficients of deviation function from the calibration of SPRT at DFPs and

$a_{i}=g_{1}\left(R_{\mathrm{TPW} 1}, \ldots, R_{\mathrm{TPWN}}, R_{\mathrm{DFP} 1}, \ldots, R_{D F P N}\right) \quad$ or $a_{i}=$ $g_{2}\left(W_{\mathrm{DFP} 1}, W_{\mathrm{DFP} 2}, \ldots, W_{D F P N}\right)$.

Matrix notation for the calculation of the coefficients of deviation function can be used. If the relationship (2) is applied to $N$ fixed points, then

$$
\left(\begin{array}{c}
\Delta W_{\mathrm{DFP} 1} \\
\vdots \\
\Delta W_{\mathrm{DFP} N}
\end{array}\right)=\left(\begin{array}{ccc}
f_{1}\left(W_{\mathrm{DFP} 1}\right) & \cdots & f_{N}\left(W_{\mathrm{DFP} 1}\right) \\
\vdots & \ddots & \vdots \\
f_{1}\left(W_{\mathrm{DFPN}}\right) & \cdots & f_{N}\left(W_{\mathrm{DFPN}}\right)
\end{array}\right)\left(\begin{array}{c}
a_{1} \\
\vdots \\
a_{N}
\end{array}\right)
$$

where $\Delta W_{\mathrm{DFP} i}=W_{\mathrm{r}, \mathrm{DFP} i}-W_{\mathrm{DFP} i}, \quad W_{\mathrm{DFP} i}$ are resistance ratios for corresponding DFP $i$ and $W_{\mathrm{r}, \mathrm{DFP} i}$ are defined in [15].

Equation (4) is written in the form

$$
\Delta \boldsymbol{W}_{\mathrm{DFP}}=\boldsymbol{M}_{\mathrm{DFP}} \boldsymbol{a}
$$

As $\boldsymbol{M}_{\mathrm{DFP}}^{-1}$ exists, coefficients of deviation function are given by

$$
\boldsymbol{a}=\boldsymbol{M}_{\mathrm{DFP}}^{-1} \Delta \boldsymbol{W}_{\mathrm{DFP}}
$$

Then the equation (2) can be rewritten as follows

$$
W_{r}=W+\boldsymbol{a}^{T} \boldsymbol{f}(\boldsymbol{W}) .
$$

\section{APPLICATION OF MONTE CARLO METHOD}

\section{A. The input data and basic relations}

Input data for Monte Carlo method are represented by SPRT resistances at defining fixed points, obtained from calibration and SPRT resistances obtained from temperature measurement. Thus, input quantities can be written as vector of dimension $2 N+2$ where

$$
\boldsymbol{R}=\left(R_{\mathrm{TPW} 1}, \ldots, R_{\mathrm{TPW} N}, R_{\mathrm{DFP} 1}, \ldots, R_{\mathrm{DFPN}}, R_{\mathrm{TPW}}, R\right)^{\mathrm{T}} .
$$

Covariance matrix and vector of input quantities will be in form $(2+2 \mathrm{~N}) \times(2+2 N)$. The resistances of SPRT are determined on the basis of SPRT calibration at DFPs, i.e. vector

$$
\boldsymbol{R}_{\mathrm{cal}}=\left(R_{\mathrm{TPW} 1}, \ldots, R_{\mathrm{TPW} N}, R_{\mathrm{DFP} 1}, \ldots, R_{\mathrm{DFP} N}\right)^{\mathrm{T}} .
$$

SPRT resistances $R$ corresponding to measured temperatures $T$, as well as SPRT resistances at triple point of 
water $R_{\mathrm{TPW}}$, their covariances and uncertainties are determined in phase of temperature measurement. It implies the vector $\boldsymbol{R}_{\text {meas }}=\left(R, R_{\mathrm{TPW}}\right)^{\mathrm{T}}$ and its covariance matrix. Beside that it is necessary to consider the covariances among SPRT resistances obtained from calibration and from temperature measurement. Then we can write the covariance matrix of the vector (8) in the form

$$
\boldsymbol{V}_{\mathrm{R}}=\left(\begin{array}{cc}
\boldsymbol{V}_{\boldsymbol{R}_{\text {meas }}} & \boldsymbol{V}_{\boldsymbol{R}_{\text {meas }}, \boldsymbol{R}_{\mathrm{cal}}} \\
\boldsymbol{V}_{\boldsymbol{R}_{\text {meas }}, \boldsymbol{R}_{\mathrm{cal}}} & \boldsymbol{V}_{\boldsymbol{R}_{\mathrm{cal}}}
\end{array}\right) .
$$

Covariance matrix $\boldsymbol{V}_{\boldsymbol{R}_{\text {meas }}}$ expresses the uncertainties of SPRT resistances and covariances among them for temperature measurements in equation (10). Covariance matrix $\boldsymbol{V}_{\boldsymbol{R}_{\text {cal }}}$ expresses uncertainties of SPRT resistances and covariances among them for calibration of SPRT at DFPs. Matrix $\boldsymbol{V}_{\boldsymbol{R}_{\text {meas }}, \boldsymbol{R}_{\text {cal }}}$ expresses the covariances among the SPRT resistances from measurement and resistances from calibration. In case of in-house temperature measurement, i.e. SPRT resistance value at TPW is used from calibration, matrix $\boldsymbol{V}_{\boldsymbol{R}_{\text {meas }}, \boldsymbol{R}_{\text {cal }}}$ has nonzero values. If we suppose that SPRT resistances during temperature measurement were obtained under the same conditions as they were in calibration process, then there could be also covariances between SPRT resistances for temperature measurement and SPRT resistances from calibration.

Usually, in practice, the covariances among SPRT resistances are not considered, excluding those at TPW. Two cases of temperature measurement are considered here, a) the use of calibrated SPRT in the laboratory (in-house), b) the use of calibrated SPRT outside the laboratory.

\section{B. Procedure of calculation}

Fig.2. schematically shows the process of calculation the temperature and its standard uncertainty by using Monte Carlo method [3].

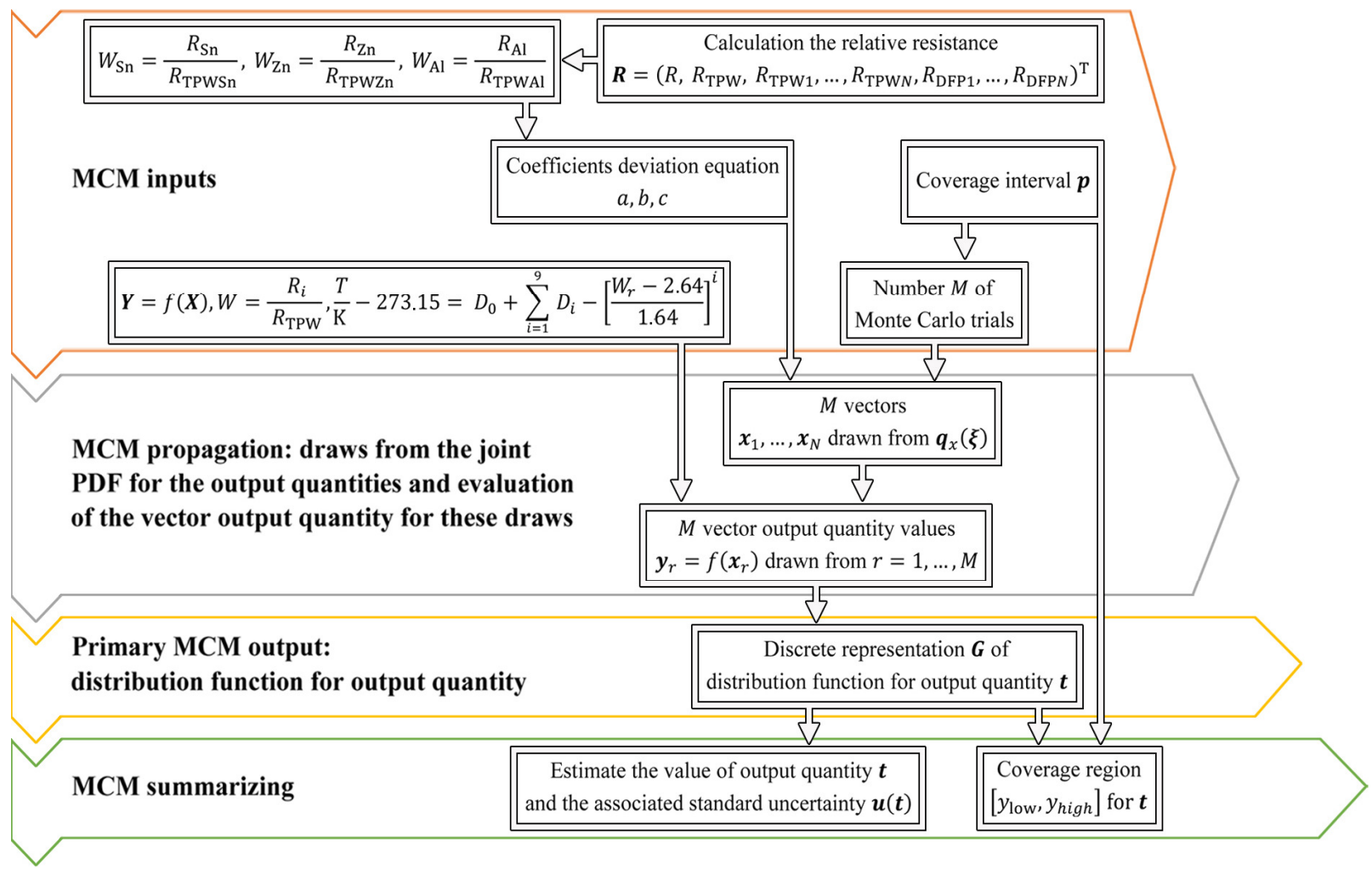

Fig.2. Computing phase of calibration using Monte Carlo method [3].

\section{Used software and pseudo-random numbers generation}

When selecting a suitable programming software environment, it was crucial to create an application which would be easy to use and portable. It was also necessary to consider the efficiency of the calculation of final application and the way of implementation of Mersenne Twister generator. For these reasons, Microsoft Visual Basic.NET programming environment was chosen and 32-bit version of operating system has been used due to direct compatibility with newer 64-bit operating systems.
Visual Basic does not integrate the generation of pseudorandom numbers with Mersenne Twister (MT) generator which is currently the best rated algorithm which has undergone a large number of experiments for testing pseudo-random numbers. Therefore, the final algorithm uses the original MT source code translated to VB.NET framework. Since MT generator generates numbers from uniform distribution, it was necessary to use the Box-Muller transformation method. This method allows transforming uniformly distributed random variables to the Gaussian 
distribution. The application of the Box-Muller transformation method can be simplified by utilizing the fact that the MT generator allows direct generation of uniformly distributed numbers over the interval $[-1,1]$. Input variables $x_{1}$ and $x_{2}$ take values from listed interval and subsequently enter formula $w=x_{1}^{2}+x_{2}^{2}$. This loop repeats until the condition $w \leq 1$ is true. Afterwards $u=\sqrt{[-2 \cdot \log (w)] / w}$ can be calculated and two independent output pseudorandom variables with normal distribution $N(0,1)$ are obtained, using $y_{1}=x_{1} \cdot u$ and $y_{2}=x_{2} \cdot u$. Normal distribution for any mean value $\mu$ and the variance $\sigma$ can be obtained as $\boldsymbol{\mu}+\boldsymbol{\sigma} \cdot \boldsymbol{z}$ where $\boldsymbol{z}$ is a matrix of randomly generated values from standard normal distribution $N(0,1)$. In our case, normal distribution is assumed for all input quantities and they are correlated in general.

If we want to construct a generator of pseudo-random numbers from a multi-dimensional normal distribution $N(\boldsymbol{\mu}, \boldsymbol{V})$, it is essential to establish dimension $n$ of multidimensional normal distribution, vector of mean values $\boldsymbol{\mu}$ of dimension $n \times 1$, covariance matrix $\boldsymbol{V}$ of dimension $n \times n$ and the number of trials that should be generated. A matrix $\boldsymbol{X}$ of dimension $n \times q$ must be generated as well. We derive $\boldsymbol{R}^{\mathrm{T}}$ from the covariance matrix $\boldsymbol{V}$ by using the Cholesky decomposition $\boldsymbol{V}=\boldsymbol{R}^{\mathrm{T}} \boldsymbol{R}$. We will generate a matrix $\boldsymbol{Z}$ of dimension $n \times q$ from the normal distribution. Then we compute $\boldsymbol{X}=\boldsymbol{\mu} \mathbf{1}^{\mathrm{T}}+\boldsymbol{R}^{\mathrm{T}} \boldsymbol{Z}$, where $\mathbf{1}$ denotes the unit vector of dimension $q$ (see [3]). The number of Monte Carlo trials $M$ that must be carried out for each sequence $h$, must be determined for calculation of the estimate of temperature $T$ and its standard uncertainty $u(T)$ by adaptive Monte Carlo method. For the output quantity $T$ we have to consider the reference probability $p$ and the number of significant decimal digits $n_{\text {dig }}$ from a standard uncertainty $u(T)$. Number of Monte Carlo trials $M$ increases as $(M \times h)$ by each further sequence of calculation $h$ to stabilize the required statistical output quantities.

\section{EVALUATION OF UNCERTAINTY AND EXPERIMENTAL DATA}

In order to compare the results of both realized cases (calibration in-house and outside the laboratory), we employ the evaluation data obtained from the Slovak Institute of Metrology (SMU) - see Table 1. These values will be used as inputs for evaluating of realization of ITS-90 and corresponding uncertainties by Monte Carlo method.

\section{A. Inputs and considered cases}

The input data are given in Table 1 . We consider the temperature measurement according to ITS-90 first in the calibration laboratory (in-house), then outside the calibration laboratory. For temperature measurement, the same TPW cell is used as was used for realization of temperature scale. In this case, the SPRT resistances at TPW after tin, zinc and aluminum are correlated (for sake of simplicity we consider the correlation coefficient $r=1$ ). SPRT resistance of temperature measurement is considered uncorrelated with the other SPRT resistances. SPRT resistances at DFPs are considered either uncorrelated (cases a), b)), or correlated (cases c), d)). The correlation coefficients among resistances at TPW and DFPs are considered uniformly $r=0.4$ while correlation coefficients among the resistances at DFPs are uniformly considered as $r=0.3$.

Case a) SPRT is used in-house, resistances at DFPs are uncorrelated

$$
\boldsymbol{R}_{R}=\left(\begin{array}{cccccccc}
1 & 1 & 1 & 0 & 0 & 0 & 1 & 0 \\
1 & 1 & 1 & 0 & 0 & 0 & 1 & 0 \\
1 & 1 & 1 & 0 & 0 & 0 & 1 & 0 \\
0 & 0 & 0 & 1 & 0 & 0 & 0 & 0 \\
0 & 0 & 0 & 0 & 1 & 0 & 0 & 0 \\
0 & 0 & 0 & 0 & 0 & 1 & 0 & 0 \\
1 & 1 & 1 & 0 & 0 & 0 & 1 & 0 \\
0 & 0 & 0 & 0 & 0 & 0 & 0 & 1
\end{array}\right)
$$$$
\boldsymbol{V}_{R}=10^{-10}\left(\begin{array}{cccccccc}
1.61 & 1.61 & 1.61 & 0 & 0 & 0 & 1.61 & 0 \\
1.61 & 1.61 & 1.61 & 0 & 0 & 0 & 1.61 & 0 \\
1.61 & 1.61 & 1.61 & 0 & 0 & 0 & 1.61 & 0 \\
0 & 0 & 0 & 14.8 & 0 & 0 & 0 & 0 \\
0 & 0 & 0 & 0 & 24.8 & 0 & 0 & 0 \\
0 & 0 & 0 & 0 & 0 & 39.9 & 0 & 0 \\
1.61 & 1.61 & 1.61 & 0 & 0 & 0 & 1.61 & 0 \\
0 & 0 & 0 & 0 & 0 & 0 & 0 & 4.00
\end{array}\right)
$$

Case b) SPRT is used outside calibration laboratory, resistances at DFPs are uncorrelated

$$
\begin{gathered}
\boldsymbol{R}_{R}=\left(\begin{array}{cccccccc}
1 & 1 & 1 & 0 & 0 & 0 & 0 & 0 \\
1 & 1 & 1 & 0 & 0 & 0 & 0 & 0 \\
1 & 1 & 1 & 0 & 0 & 0 & 0 & 0 \\
0 & 0 & 0 & 1 & 0 & 0 & 0 & 0 \\
0 & 0 & 0 & 0 & 1 & 0 & 0 & 0 \\
0 & 0 & 0 & 0 & 0 & 1 & 0 & 0 \\
0 & 0 & 0 & 0 & 0 & 0 & 1 & 0 \\
0 & 0 & 0 & 0 & 0 & 0 & 0 & 1
\end{array}\right), \\
\boldsymbol{V}_{R}=10^{-10}\left(\begin{array}{cccccccc}
1.61 & 1.61 & 1.61 & 0 & 0 & 0 & 0 & \\
1.61 & 1.61 & 1.61 & 0 & 0 & 0 & 0 & 0 \\
1.61 & 1.61 & 1.61 & 0 & 0 & 0 & 0 & 0 \\
0 & 0 & 0 & 14.8 & 0 & 0 & 0 & 0 \\
0 & 0 & 0 & 0 & 24.8 & 0 & 0 & 0 \\
0 & 0 & 0 & 0 & 0 & 39.9 & 0 & 0 \\
0 & 0 & 0 & 0 & 0 & 0 & 1.61 & 0 \\
0 & 0 & 0 & 0 & 0 & 0 & 0 & 4.00
\end{array}\right)
\end{gathered}
$$

Case c) SPRT is used in-house, resistances at DFPs are correlated

$$
\begin{gathered}
\boldsymbol{R}_{R}=\left(\begin{array}{cccccccc}
1 & 1 & 1 & 0.4 & 0.4 & 0.4 & 1 & 0 \\
1 & 1 & 1 & 0.4 & 0.4 & 0.4 & 1 & 0 \\
1 & 1 & 1 & 0.4 & 0.4 & 0.4 & 1 & 0 \\
0.4 & 0.4 & 0.4 & 1 & 0.3 & 0.3 & 0.4 & 0 \\
0.4 & 0.4 & 0.4 & 0.3 & 1 & 0.3 & 0.4 & 0 \\
0.4 & 0.4 & 0.4 & 0.3 & 0.3 & 1 & 0.4 & 0 \\
1 & 1 & 1 & 0.4 & 0.4 & 0.4 & 1 & 0 \\
0 & 0 & 0 & 0 & 0 & 0 & 0 & 1
\end{array}\right), \\
\boldsymbol{V}_{R}=10^{-10}\left(\begin{array}{ccccccccc}
1.61 & 1.61 & 1.61 & 1.96 & 2.53 & 3.21 & 1.61 & 0 \\
1.61 & 1.61 & 1.61 & 1.96 & 2.53 & 3.21 & 1.61 & 0 \\
1.61 & 1.61 & 1.61 & 1.96 & 2.53 & 3.21 & 1.61 & 0 \\
1.96 & 1.96 & 1.96 & 14.8 & 5.75 & 7.30 & 1.96 & 0 \\
2.53 & 2.53 & 2.53 & 5.75 & 24.8 & 9.44 & 2.53 & 0 \\
3.21 & 3.21 & 3.21 & 7.30 & 9.44 & 39.9 & 3.21 & 0 \\
1.61 & 1.61 & 1.61 & 1.96 & 2.53 & 3.21 & 1.61 & 0 \\
0 & 0 & 0 & 0 & 0 & 0 & 0 & 4.00
\end{array}\right)
\end{gathered}
$$


Case d) SPRT is used outside calibration laboratory, resistances at DFPs are correlated

$$
\begin{gathered}
\boldsymbol{R}_{R}=\left(\begin{array}{cccccccc}
1 & 1 & 1 & 0.4 & 0.4 & 0.4 & 0 & 0 \\
1 & 1 & 1 & 0.4 & 0.4 & 0.4 & 0 & 0 \\
1 & 1 & 1 & 0.4 & 0.4 & 0.4 & 0 & 0 \\
0.4 & 0.4 & 0.4 & 1 & 0.3 & 0.3 & 0 & 0 \\
0.4 & 0.4 & 0.4 & 0.3 & 1 & 0.3 & 0 & 0 \\
0.4 & 0.4 & 0.4 & 0.3 & 0.3 & 1 & 0 & 0 \\
0 & 0 & 0 & 0 & 0 & 0 & 1 & 0 \\
0 & 0 & 0 & 0 & 0 & 0 & 0 & 1
\end{array}\right), \\
\boldsymbol{V}_{R}=10^{-10}\left(\begin{array}{ccccccccc}
1.61 & 1.61 & 1.61 & 1.96 & 2.53 & 3.21 & 0 & \\
1.61 & 1.61 & 1.61 & 1.96 & 2.53 & 3.21 & 0 & 0 \\
1.61 & 1.61 & 1.61 & 1.96 & 2.53 & 3.21 & 0 & 0 \\
1.96 & 1.96 & 1.96 & 14.8 & 5.75 & 7.30 & 0 & 0 \\
2.53 & 2.53 & 2.53 & 5.75 & 24.8 & 9.44 & 0 & 0 \\
3.21 & 3.21 & 3.21 & 7.30 & 9.44 & 39.9 & 0 & 0 \\
0 & 0 & 0 & 0 & 0 & 0 & 1.61 & 0 \\
0 & 0 & 0 & 0 & 0 & 0 & 0 & 4.00
\end{array}\right)
\end{gathered}
$$

Whereby $\boldsymbol{V}_{R}=\boldsymbol{P}_{R} \boldsymbol{R}_{R} \boldsymbol{P}_{R}^{\mathrm{T}}$ and $\boldsymbol{P}_{R}$ is a diagonal matrix of dimension $8 \times 8$ with diagonal elements:

$u\left(R_{\mathrm{TPW} S \mathrm{n}}\right), u\left(R_{\mathrm{TPW} \mathrm{Zn}}\right), u\left(R_{\mathrm{TPW} \mathrm{Al}}\right), u\left(R_{\mathrm{Sn}}\right), u\left(R_{\mathrm{Zn}}\right)$, $u\left(R_{\mathrm{Al}}\right), u\left(R_{\mathrm{TPW}}\right), u(R)$.

The results of simulation by Monte Carlo method and GUM are presented in Table 4. The graphical comparison of both methods for 66 calibration points within the range $(0 \div$ $660){ }^{\circ} \mathrm{C}$ of the ITS-90 is illustrated in Fig. 10.

Table 1. Measured values of SPRT resistances in defining fixed point.

\begin{tabular}{ccc}
\hline $\begin{array}{c}\text { Defining fixed } \\
\text { point }\end{array}$ & $\begin{array}{c}\text { Resistance } \\
(\Omega)\end{array}$ & $\begin{array}{c}\text { Standard uncertainty of } \\
\text { resistance }(\Omega)\end{array}$ \\
\hline $\mathrm{Sn}$ & 46.9397533 & $3.85 \times 10^{-5}$ \\
$\mathrm{Zn}$ & 63.7056752 & $4.98 \times 10^{-5}$ \\
$\mathrm{Al}$ & 83.7191875 & $6.32 \times 10^{-5}$ \\
$\mathrm{TPW}_{\mathrm{Sn}}$ & 24.8002001 & $1.27 \times 10^{-5}$ \\
TPW $_{\mathrm{Zn}}$ & 24.8001927 & $1.27 \times 10^{-5}$ \\
TPW $_{\mathrm{Al}}$ & 24.8001872 & $1.27 \times 10^{-5}$ \\
\hline
\end{tabular}

We consider $M=10^{5}$ Monte Carlo trials. For the output quantity $T$ we consider the reference probability $p=0.95$ and the number of significant decimals of the standard uncertainty $n_{\text {dig }}=2$. Fig.3. shows a histogram of resistance $R_{\mathrm{Al}}$, as given in the example 5.B.

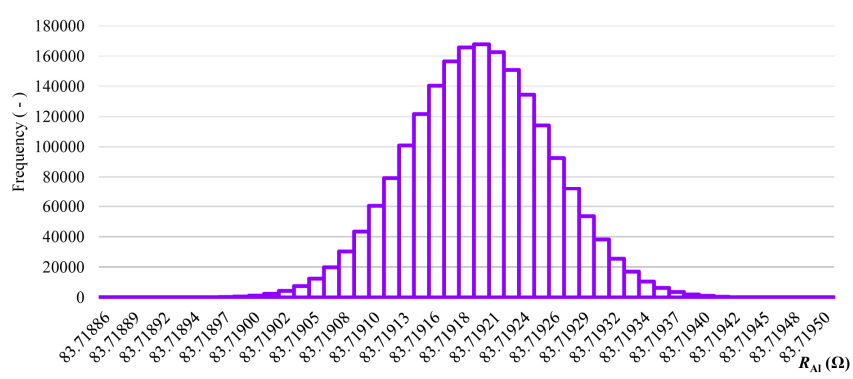

Fig.3. Histogram of input resistance.

The coefficients $a, b, c$ of deviation function can be determined from equation (6), see Fig.5. for their calculation. Histograms of coefficients of deviation function and the coefficient $a$, presented in Fig.4., have similar shape.

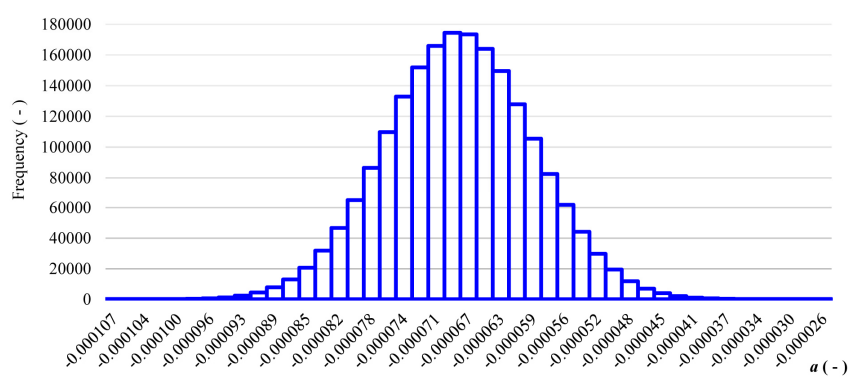

Fig.4. Histogram of the deviation equation for coefficient $a$.

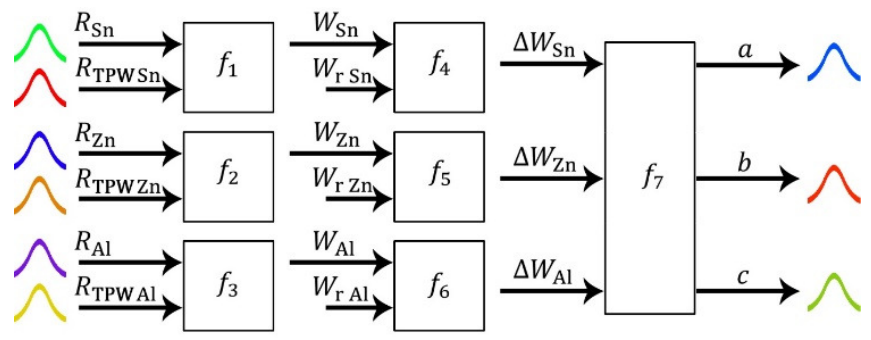

Fig.5. Sub-model for calculation of the coefficients of deviation function.

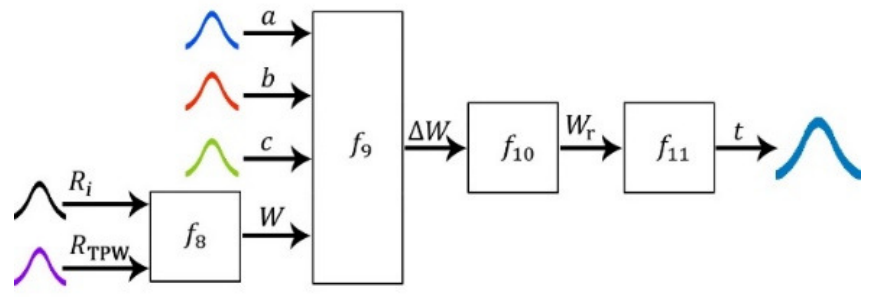

Fig.6. Model to calculate temperature and corresponding standard uncertainty.

Fig.6. shows the calculation procedure for determination of the temperature $T$, where $f_{8}=\frac{R}{R_{\mathrm{TPW}}}$, from equation (2) we can determine $f_{9}=a(W-1)+b(W-1)^{2}+$ $c(W-1)^{3}$ and from equation (7) we get $f_{10}=W-$ $a(W-1)+b(W-1)^{2}+c(W-1)^{3}$. Histogram of estimated temperature $t$ is presented in Fig.8.

\section{B. Example of calculating the output characteristics for the case b)}

Temperature according to ITS-90 and corresponding standard uncertainty was determined for data in Table 1. and for correlation matrix of resistances for case b, see Table 2 .

Table 2. Evaluation of specific resistance using the law of propagation of uncertainty.

\begin{tabular}{ccc}
\hline$R_{i}(\Omega)$ & $t_{i}\left({ }^{\circ} \mathrm{C}\right)$ & $u\left(t_{i}\right)\left({ }^{\circ} \mathrm{C}\right)$ \\
\hline 46.55489887 & 227.75076 & $5.59864 \times 10^{-4}$ \\
\hline
\end{tabular}


Let's use the MCM with $h=20$ and $M=10^{5}$. Based on the generated input values for resistances and using appropriate relationship, we get estimate of the temperature $t\left({ }^{\circ} \mathrm{C}\right)$.

$$
t=\left[\begin{array}{c}
227.7479428 \\
\vdots \\
227.7533240
\end{array}\right]
$$

The symmetrical reference interval with the specified probability for the estimating output quantity $t$ is obtained from its generated discrete representation (distribution function shown in Fig.7.). To do so, generated values are arranged at non-decreasing sequence, using rules listed in [3]. According to that procedure, we get the following symmetric confidence interval

$$
t=\left[y_{\min } ; y_{\max }\right]=[227.74966142 ; 227.75185372]
$$

and following narrowest confidence interval

$$
t=\left[y_{\min } ; y_{\max }\right]=[227.74966148 ; 227.75185375]
$$

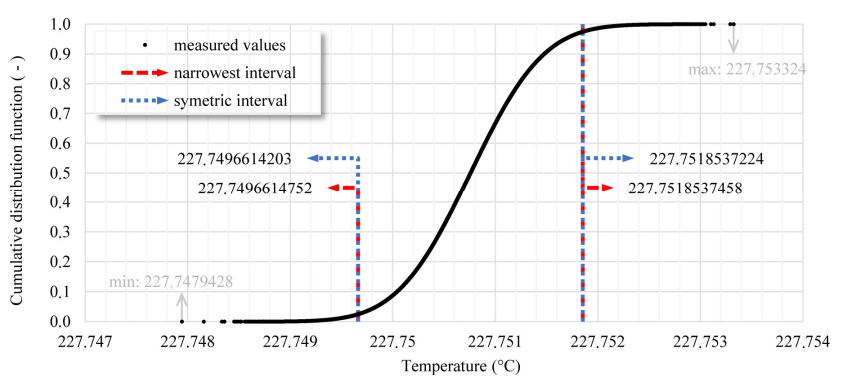

Fig.7. The distribution function of the output temperature.

Statistical characteristics of the resulting estimates are calculated from the partial estimates in each sequence $y^{(h)}$, $u\left(y^{(h)}\right)$. After calculating the last sequence $h$, it is possible to calculate the resulting parameters for estimation. In this case

$$
\begin{gathered}
\hat{y}=y=\frac{1}{h} \sum_{i=1}^{h} y^{(i)}=227.7507574{ }^{\circ} \mathrm{C} \\
s_{\hat{y}}=\sqrt{\frac{1}{h(h-1)} \sum_{i=1}^{h}\left(y^{(i)}-\hat{y}\right)^{2}}=3.39088 \times 10^{-7}{ }^{\circ} \mathrm{C}
\end{gathered}
$$

The standard uncertainty

$$
\begin{gathered}
\hat{u}(y)=\frac{1}{h} \sum_{i=1}^{h} u\left(y^{(i)}\right)=5.59883 \times 10^{-4} \\
s_{\widehat{u}(y)}=\sqrt{\frac{1}{h(h-1)} \sum_{i=1}^{h}\left(u\left(y^{(i)}\right)-\hat{u}(y)\right)^{2}}=2.70938 \times \\
10^{-7}{ }^{\circ} \mathrm{C}
\end{gathered}
$$

The lower end point of the narrowest confidence interval of stabilization criteria

$$
\begin{gathered}
\hat{y}_{\min }=\frac{1}{h} \sum_{i=1}^{h} y_{\min }^{(i)}=\frac{1}{20}\left(y_{\min }^{(1)}+y_{\min }^{(2)}+\ldots+y_{\min }^{(20)}\right)= \\
227.7496603{ }^{\circ} \mathrm{C}
\end{gathered}
$$

The upper end point of the narrowest confidence interval of stabilization criteria

$$
\begin{gathered}
\hat{y}_{\max }=\frac{1}{h} \sum_{i=1}^{h} y_{\max }^{(i)}=\frac{1}{20}\left(y_{\min }^{(1)}+y_{\min }^{(2)}+\ldots+y_{\min }^{(20)}\right)= \\
227.7518512{ }^{\circ} \mathrm{C}
\end{gathered}
$$

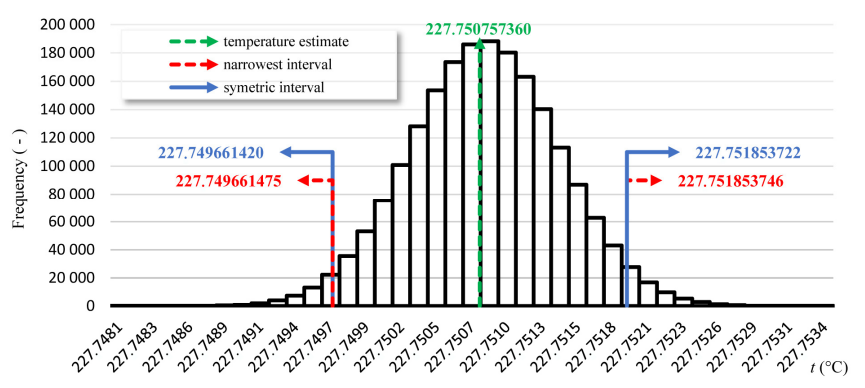

Fig.8. Histogram of output quantity $t$.

Numerical tolerance $\delta$ (see more in 5.C.), expressed by the standard uncertainty $\hat{u}(y)$, is

$$
\delta=\frac{1}{2} \times 10^{-5}=0.000005=5 \times 10^{-6}
$$

Stabilization criterion determines whether it is necessary to increase the current value of the sequence $h>2$ calculation of Monte Carlo method to the next sequence. This is the case if one of values $2 s_{\hat{y}}, 2 s_{\hat{u}(y)}, 2 s_{\hat{y}_{\text {min }}}$ and $2 s_{\hat{y}_{\max }}$ becomes greater than $\delta$. If the stabilization criteria are successfully met, $y^{(h \times M)}, u\left(y^{(h \times M)}\right)$ are final statistical characteristics and $\left[y_{\text {low }}^{(h \times M)} ; y_{\text {high }}^{(h \times M)}\right]$ are determined from all generated values. In the given example, following results were obtained by the Monte Carlo method:

Estimation of the temperature $t=227.75075736{ }^{\circ} \mathrm{C}$, standard uncertainty $u(t)=5.59884 \times 10^{-4}{ }^{\circ} \mathrm{C}, \quad 95 \%$ narrowest interval [227.74966148;227.75185375] and $95 \%$ symmetric interval [227.74966142;227.75185372]. Statistical characteristic of output variable $t$, obtained by the adaptive Monte Carlo method, is shown in Fig.8. as a graph of probability distribution, where the model with one output variable $t$ enters the submodel with multiple output variables $a, b, c$. 


\section{Validation of law of propagation of uncertainty}

Calculation should prove if the reference interval obtained by law of propagation of uncertainty and by Monte Carlo method is identical in certain numerical tolerance. This numerical tolerance is assessed in relation to end points of the reference interval and it gives expression of standard uncertainty $u(y)$ to the existing number of decimal places. Numerical expression of tolerance $\delta$ with an associated standard uncertainty $u(y)$, as described in section 7.9.2 [4], is $\delta=\frac{1}{2} \times 10^{r}, u(y)=56 \cdot 10^{-5}{ }^{\circ} \mathrm{C}, a=56, r=-5$,

$$
\Rightarrow \quad \delta=\frac{1}{2} \cdot 10^{-5}=0,00005^{\circ} \mathrm{C}
$$

Absolute differences of limit values of both confidence intervals are determined as

$$
\begin{aligned}
& d_{\text {low }}=\left|y_{\mathrm{GUM}}-U_{0,95(\mathrm{GUM})}-y_{\text {low(MCM) }}\right| \\
& d_{\text {high }}=\left|y_{\mathrm{GUM}}+U_{0,95(\mathrm{GUM})}-y_{\text {high(MCM) }}\right|
\end{aligned}
$$

Table 4. shows detailed result of uncertainty of temperature measurement according to ITS 90 for each case listed. The maximum number of sequences needed for validation of each case was limited to 250 . Table 3 . contains validation results and minimum necessary number of sequences for all cases.

Fig.9. shows the user interface of created application for the evaluation and validation of the standard platinum resistance thermometer by the Monte Carlo method.
Table 3. Min. number of sequences $h$ (each from $M=10^{5}$ ) and

\begin{tabular}{|c|c|c|c|c|c|}
\hline$n$ & $R i(\Omega)$ & $\begin{array}{c}\text { Case } \\
\text { a) }\end{array}$ & b) & c) & d) \\
\hline 34 & 454.7129134 & & $41 \checkmark$ & & \\
\hline 35 & 555.6193595 & & $45 \checkmark$ & & \\
\hline 36 & 656.5258056 & & $30 v$ & & \\
\hline 37 & 757.4322516 & & $20 r$ & & \\
\hline 38 & 858.3386977 & & $54 \checkmark$ & & \\
\hline 39 & 959.2451438 & & $43 r$ & & \\
\hline 40 & 060.1515898 & & $50 r$ & & \\
\hline 41 & 61.0580359 & & $106 \checkmark$ & & \\
\hline 42 & 61.9644820 & & $66 r$ & & \\
\hline 43 & 62.8709280 & & $45 \checkmark$ & & \\
\hline 44 & 63.7773741 & & $56 \checkmark$ & & \\
\hline 45 & 64.6838202 & & $59 \checkmark$ & & \\
\hline 46 & 65.5902662 & & $73 r$ & & \\
\hline 47 & 66.4967123 & & $46 \checkmark$ & & \\
\hline 48 & 67.4031583 & & $117 \checkmark$ & & \\
\hline 49 & 68.3096044 & & $58 r$ & & $250 \times$ \\
\hline 50 & 69.2160505 & $250 x$ & $76 r$ & $250 x$ & \\
\hline 51 & 70.1224965 & & $85 \checkmark$ & & \\
\hline 52 & 71.0289426 & & $101 \checkmark$ & & \\
\hline 53 & 71.9353887 & & $79 \checkmark$ & & \\
\hline 54 & 472.8418347 & & $66 r$ & & \\
\hline 55 & 73.7482808 & & $82 \checkmark$ & & \\
\hline 56 & 74.6547269 & & $85 v$ & & \\
\hline 57 & 75.5611729 & & $61 \checkmark$ & & \\
\hline 58 & 76.467619 & & $250 x$ & & \\
\hline 59 & 77.3740651 & & $89 \checkmark$ & & \\
\hline 60 & 78.2805111 & & $82 \checkmark$ & & \\
\hline 61 & 79.1869572 & & $128 v$ & & \\
\hline 62 & 80.0934032 & & $89 \checkmark$ & & \\
\hline 63 & 80.9998493 & & $151 \checkmark$ & & \\
\hline 64 & 81.9062954 & & $2 v$ & & \\
\hline 65 & 82.8127414 & & $2 v$ & & $124 \checkmark$ \\
\hline 66 & 83.7191875 & & $2 r$ & & $124 \checkmark$ \\
\hline
\end{tabular}
validation result (yes $\checkmark$, no $\mathbf{x}$ ).

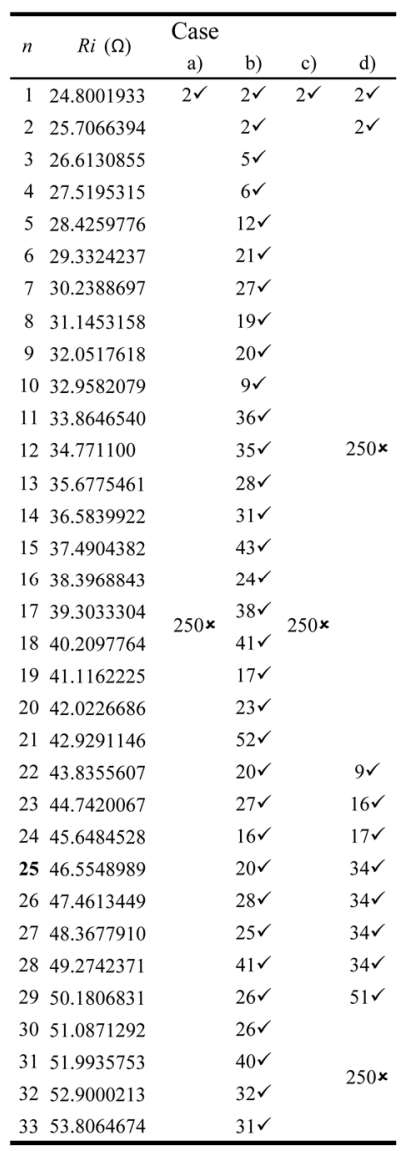

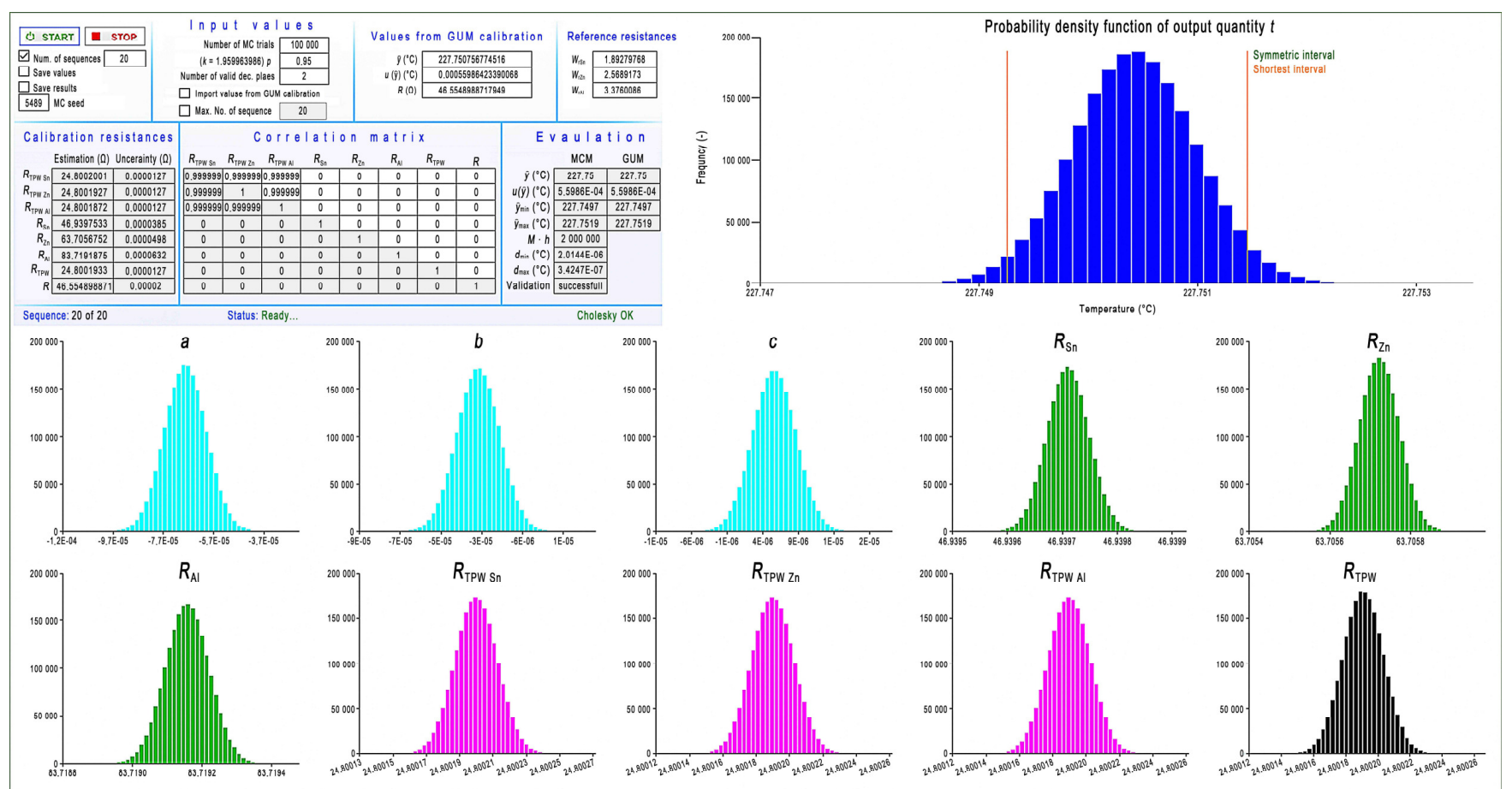

Fig.9. User interface of the application at the end of calculation. 
Table 4. Comparison and verification of the law of uncertainty propagation through the law of distribution propagation using MCM for one value $t$ in different cases ( $\checkmark=$ validation successful, $\boldsymbol{x}=$ validation unsuccessful).

\begin{tabular}{|c|c|c|c|c|c|c|}
\hline Case & Method & $\underset{\left(\times 10^{5}\right)}{M}$ & $\begin{array}{c}t \\
\left({ }^{t} \mathrm{C}\right)\end{array}$ & $\underset{\left({ }^{\circ} \mathrm{C} \times 10^{-4}\right)}{\boldsymbol{u}(t)}$ & $\begin{array}{l}95 \% \text { coverage } \\
\text { interval } \\
\text { Low } \\
\text { High }\end{array}$ & $\begin{array}{c}\text { GUF } \\
\text { validated }\end{array}$ \\
\hline \multirow[t]{3}{*}{ a) } & GUF & - & 227.750757 & 4.24 & $\begin{array}{l}227.749925 \\
227.751588\end{array}$ & - \\
\hline & $\begin{array}{l}\text { MCM } \\
\text { shortest }\end{array}$ & 1 & 227.750758 & 4.98 & $\begin{array}{l}227.749769 \\
227.751722\end{array}$ & $x$ \\
\hline & $\begin{array}{l}\text { MCM } \\
\text { shortest } \\
\text { MCM } \\
\text { symmetric }\end{array}$ & 250 & 227.750757 & 5.60 & $\begin{array}{l}227.749657 \\
227.751852 \\
227.749660 \\
227.751855 \\
\end{array}$ & $\begin{array}{l}x \\
x\end{array}$ \\
\hline \multirow[t]{3}{*}{ b) } & GUF & - & 227.750757 & 5.60 & $\begin{array}{l}227.749659 \\
227.751854\end{array}$ & - \\
\hline & $\begin{array}{l}\text { MCM } \\
\text { shortest }\end{array}$ & 1 & 227.750758 & 5.60 & $\begin{array}{l}227.749672 \\
227.751863\end{array}$ & $x$ \\
\hline & $\begin{array}{l}\text { MCM } \\
\text { shortest } \\
\text { MCM } \\
\text { symmetric }\end{array}$ & 20 & 227.750757 & 5.60 & $\begin{array}{l}227.749661 \\
227.751854 \\
227.749661 \\
227.751854 \\
\end{array}$ & $\begin{array}{l}\checkmark \\
\checkmark\end{array}$ \\
\hline \multirow[t]{3}{*}{ c) } & GUF & - & 227.750757 & 4.21 & $\begin{array}{l}227.749931 \\
227.751583\end{array}$ & - \\
\hline & $\begin{array}{l}\text { MCM } \\
\text { shortest }\end{array}$ & 1 & 227.750758 & 4.76 & $\begin{array}{l}227.749823 \\
227.751687\end{array}$ & $x$ \\
\hline & $\begin{array}{l}\text { MCM } \\
\text { shortest } \\
\text { MCM } \\
\text { symmetric }\end{array}$ & 250 & 227.750757 & 4.75 & $\begin{array}{l}227.749831 \\
227.751692 \\
227.749827 \\
227.751688 \\
\end{array}$ & $\begin{array}{l}x \\
x\end{array}$ \\
\hline \multirow[t]{3}{*}{ d) } & GUF & - & 227.750757 & 4.75 & $\begin{array}{l}227.749826 \\
227.751687\end{array}$ & - \\
\hline & $\begin{array}{l}\text { MCM } \\
\text { shortest }\end{array}$ & 1 & 227.750758 & 4.76 & $\begin{array}{l}227.749823 \\
227.751687\end{array}$ & $\checkmark$ \\
\hline & $\begin{array}{l}\text { MCM } \\
\text { shortest } \\
\text { MCM } \\
\text { symmetric }\end{array}$ & 34 & 227.750757 & 4.75 & $\begin{array}{l}227.749829 \\
227.751690 \\
227.749827 \\
227.751688 \\
\end{array}$ & $\begin{array}{l}\checkmark \\
\checkmark\end{array}$ \\
\hline \multirow[t]{3}{*}{ e) } & GUF & - & 227.750757 & 4.22 & $\begin{array}{l}227.749929 \\
227.751585\end{array}$ & - \\
\hline & $\begin{array}{l}\text { MCM } \\
\text { shortest }\end{array}$ & 1 & 227.750758 & 4.98 & $\begin{array}{l}227.749769 \\
227.751722\end{array}$ & $x$ \\
\hline & $\begin{array}{l}\text { MCM } \\
\text { shortest } \\
\text { MCM } \\
\text { symmetric }\end{array}$ & 250 & 227.750757 & 4.98 & $\begin{array}{l}227.749781 \\
227.751732 \\
227.749782 \\
227.751733 \\
\end{array}$ & $x$ \\
\hline \multirow[t]{3}{*}{ f) } & GUF & - & 227.750757 & 4.98 & $\begin{array}{l}227.749781 \\
227.751732\end{array}$ & - \\
\hline & $\begin{array}{l}\text { MCM } \\
\text { shortest }\end{array}$ & 1 & 227.750758 & 4.98 & $\begin{array}{l}227.749769 \\
227.751722\end{array}$ & $x$ \\
\hline & $\begin{array}{l}\text { MCM } \\
\text { shortest } \\
\text { MCM } \\
\text { symmetric }\end{array}$ & 82 & 227.750757 & 4.98 & $\begin{array}{l}227.749778 \\
227.751729 \\
227.749782 \\
227.751733 \\
\end{array}$ & $\begin{array}{l}\checkmark \\
\checkmark\end{array}$ \\
\hline
\end{tabular}

GUM uncertainty framework (GUF) [1], each sequence of MCM consist of $\mathrm{M}=1 \times 10^{5}$ trials

\section{CONCLUSIONS}

This paper presents a procedure employing the Monte Carlo method for the determination of uncertainties of temperature scale. The procedure is based on generating pseudo-random numbers for the input SPRT resistances at DFPs and at TPW.

In order to consider the correlations among DFPs, the approach of generating pseudo-random numbers from multivariate distributions was used. To do so, an 8dimensional Gaussian probability distribution was assumed. The assumption of Gaussian distribution is quite acceptable, given several sources of uncertainty of SPRT resistances at DFPs. If the correlations between the SPRT resistances at DFPs are negligible, it is possible to adapt the model so that the input resistances are uncorrelated and one-dimensional distributions for each input resistance can be used.

Fig.10. shows the course of uncertainty for the entire range considered and for each case. The figure compares uncertainties of temperatures obtained by using MCM and by law of propagation of uncertainty. As already mentioned in [16], the uncertainty due to the correlation between resistances of SPRT at DFPs can reduce the value of the uncertainty of temperature, doing so even in DFPs themselves.

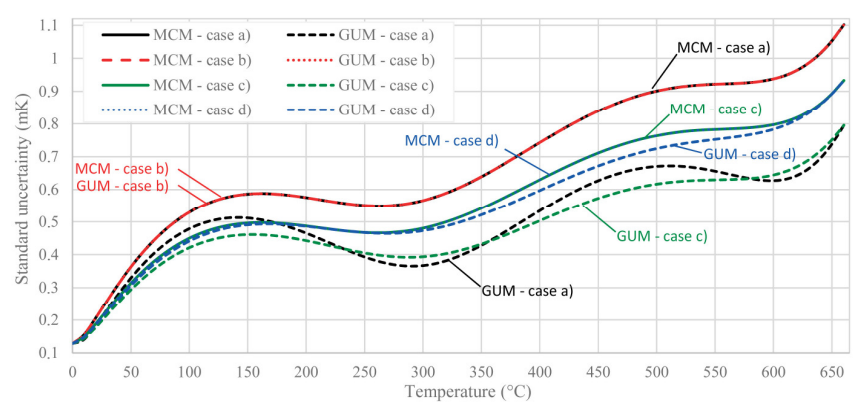

Fig.10. Comparison of MCM and GUM for different cases, illustrated on subrange $(0 \div 660){ }^{\circ} \mathrm{C}$ of ITS-90.

Attention was also paid to validation of the use of law of propagation of uncertainty in accordance with the GUM for particular conditions. On the basis of validation, we found out that for some cases the results given by using MCM and law of propagation of uncertainty were not consistent.

\section{ACKNOWLEDGMENT}

Authors would like to thank the Slovak University of Technology in Bratislava, the APVV grant agency, projects number APVV15-0295, APVV 15-0164; the VEGA grant agency, projects number $1 / 0604 / 15,1 / 0748 / 15$; and the KEGA agency, projects number 014STU-4/2015 and 039STU-4/2017 for their support.

\section{REFERENCES}

[1] Joint Committee for Guides in Metrology. (2008). Evaluation of measurement data - Guide to the expression of uncertainty in measurement. JCGM 100:2008.

[2] White, D.R., Ballico, M., Chimenti, V., et al. (2009). CCT Working Document CCT/08-19/rev.

[3] Joint Committee for Guides in Metrology. (2008). Evaluation of measurement data - Supplement 1: "Guide to the expression of uncertainty in measurement" - Propagation of distributions using a Monte Carlo method. JCGM 101:2008. 
[4] Joint Committee for Guides in Metrology. (2011). Evaluation of measurement data - Supplement 2: "Guide to the expression of uncertainty in measurement" - Extension to any number of output quantities. JCGM 102:2011.

[5] Rosenkranz, P. (2011). Uncertainty propagation for platinum resistance thermometers calibrated according to ITS-90. International Journal of Thermophysics, 32 (1), 106-119.

[6] Duris, S., Palencar, R., Ranostaj, J. (2008). The efect of covariance on uncertainty when constructing the ITS-90 temperature scale. Measurement Techniques, 51 (4), 412-420.

[7] Duris, S., Palencar, R. (2006). A matrix interpretation of the estimate of the extension of uncertainties when constructing a temperature scale. Measurement Techniques, 49 (7), 689-696.

[8] Witkovsky, V. (2013). On exactmultiple-use linear calibration confidence intervals. In MEASUREMENT 2013 : 9th International Conference on Measurement. Bratislava, Slovakia : Institute of Measurement Science SAS, 35-38.

[9] Lira, I., Grientschnig, D. (2013). A formalism for expressing the probability density functions of interrelated quantities. Measurement Science Review, 13 (2), 50-55.

[10] Kosarevsky, S.V., Latypov, V.N. (2012). Practical procedure for position tolerance uncertainty determination via Monte-Carlo error propagation. Measurement Science Review, 12 (1), 1-7.

[11] Zhang, F. Qu, X. (2012). Fusion estimation of point sets from multiple stations of spherical coordinate instruments utilizing uncertainty estimation based on Monte Carlo. Measurement Science Review, 12 (2), 40-45.

[12] Ribeiro, S., Alves, J., Oliveira, C., Pimenta, M., Cox, M.G. (2008). Uncertainty evaluation and validation of a comparison methodology to perform in-house calibration of platinum resistance thermometers using a Monte Carlo method. International Journal of Thermophysics, 29 (3), 902-914.

[13] Palenčár, R., Ďuriš, S., Dovica, M., Palenčár, J. (2015). Application of Monte Carlo method for evaluation of uncertainties of temperature measurement by SPRT. In XXI IMEKO World Congress "Measurement in Research and Industry". IMEKO, 6 p.

[14] Lira, I. Grientschnig, D. (2015). Bayesian analysis of a simple measurement model distinguishing between types of information. Measurement Science Review, 15 (6), 274-283.

[15] Preston-Thomas, H. (1990). The International temperature scale of 1990 (ITS-90). Metrologia, 27 (1), 3-10.

[16] Palencar, R., Duris, S., Durisova, Z., Brokes, V., Pavlasek, P. (2016). Reduction of measurement uncertainty by taking into account correlation in measurements and temperature scale realization. In Measurement Techniques, 59 (1), 52-58.

Received December 12, 2016. Accepted May 09, 2017. 